kỳ $(83,3 \%)$.

- Có mối liên quan giữa mức độ thiếu máu với tình trạng dinh dưỡng của bệnh nhân.

\section{TÀI LIÊU THAM KHẢO}

1. Hội Tiết niệu Thận học Việt Nam (2013), Chẩn đoán và đánh giá thiếu máu trong bệnh thân mạn, Hướng dẫn điêu trị thiếu máu trong bệnh thần man. tr. 10-11.

2. Bạch Quốc Tuyên (2002), Đại cương về thiếu máu, Bách khoa thư bệnh học. Tập 1, Nhà xuất bản Y học.

3. Hà Thi Thanh (2009), Chương huyết học cở sở. Huyết học - truyên máu. Nhà xuất bản $Y$ học.

4. Alemu B, et al (2021), Prevalence of Anemia and Its Associated Factors Among Chronic Kidney Disease Patients Attending Selected Public Hospitals of Addis Ababa, Ethiopia: InstitutionalBased Cross-Sectional Study. Original Research. 14-2021: p. 67-75.
5. Bárány $\mathbf{P}$, et al (1991), Nutritional assessment in anemic hemodialysis patients treated with recombinant human erythropoietin. Clin Nephrol. 35(6): p. 370-379.

6. Fliss E. M. Murtagh, J.A.H., Irene J.Higginson (2007), The prevalence of symptoms in ERSD: a systematic review. Advances in CKD-the journal of the national kidney foundation. 14(1): p. 82-99.

7. Kammerer J, et al (2002), Anemia in CKD: prevalence, diagnosis and treatment. Case study of the anemic patient. Nephrol nursing journal. 29(4): p. 371-374.

8. Lynsey Webb, et al (2009), Demographic and biochemistry profile of kidney transphant rcipients in the $\mathrm{U} \mathrm{K}$ in 2008: national and centre-specific analyses, The 12th Annual report from the UK renal registry. p. 69-102.

9. Maria Amélia Aguiar Hazin (2020), Anemia in chronic kidney disease. Revista da Associação Médica Brasileira. 66.

\title{
SẢN XUẤT THUỐC BẰNG CÔNG NGHÊ IN 3D FDM PHẦN 1: NGUYÊN LÝ VÀ QUY TRÌNH HOẠT ĐộNG
}

\section{TÓM TẮT}

In 3D là công nghệ tạo vật thể 3 chiều có nhiều triển vọng trong việc bào chế thuốc cá nhân hóa đáp ứng nhu câu điều trị riêng biệt cùng từng người bệnh. Công ghệ này đồng thời cho phép bào chế các dạng thuốc với liêu lượng chính xác, có cấu trúc phức tạp kiểm soát giải phóng chỉ bằng một vài cái nhấp chuột máy tính. Bài tổng quan này trình bày một cách có hệ thống nguyên lý cấu tạo, cơ chế hoạt động của máy in 3D ứng dụng phổ biến trong nghành Dược. Bên cạnh đó, bài viết mô tả chi tiết quy trình từng bước tạo ra một vật thể bằng kỹ thuật in $3 \mathrm{D}$ dựa trên nguyền lý FDM (fused deposition modeling).

Tứ khóa: Công nghệ in 3D, thuốc in 3D, cá nhân hóa điều trị.

\section{SUMMARY}

\section{APPLICATION OF 3D FDM IN \\ PHARMACEUTICAL FORMULATION DESIGN: OPERATION FUNDAMETAL AND TECHNICAL CHALLENGES}

3 dimension (3D) printing was utilized, for the first time, as a manufacturing platform for fabrication of pharmaceutical dosage forms in 2000 [1]. However, not until the first approval of Spritam by US. FDA in 2015, application of 3D printing in pharmaceutical

*Trường đại hoc Dước Hà Nôi

Chịu trách nhiệm chính: Võ Quốc Ánh

Email: anhvq@hup.edu.vn

Ngày nhận bài: 13.4.2021

Ngày phản biên khoa học: 27.5.2021

Ngày duyệt bài: 11.6.2021
Lê Thị Thu Trang*, Trần Thị Hải Yến*, Nguyễn Thị Thanh Duyên*, Võ Quốc Ánh*

sciences gained its growing interest. The technology is involving in the layer-by-layer deposition of materials to fabricate 3 dimension objects according to a digital model. The American Society for Testing and Materials (ASTM) classified 3D printing technology into 7 categories, namely material extrusion, material jetting, powder bed fusion, binder jetting, vat photopolymerisation, sheet lamination and directed energy deposition. 3D fused deposition modelling (FDM), a material extrusion based technique, is the most researched technique owing to its simple, low cost and highly flexibility. FDM allows to fabricate various dosage forms with highly complex geometries that offer a great potential to customize drug release profiles. This paper reviewed systematically the technical fundamental, requirements, step by step procedure and critical parameters of a 3D FDM process.

Keywords: Three-dimension (3D) printing, 3D printed drugs, fused deposition modeling, material extrusion.

\section{I. ĐẶT VẤN ĐỀ}

In 3D là một một nền tảng công nghệ mới ứng dụng trong nhiều lĩnh vực sản xuất và công nghiệp khác nhau. Ứng dụng công nghệ in 3D trong nghiên cứu bào chế và sản xuất dược phẩm nhận được nhiều sự quan tâm trong gần một thập kỷ gần đây, đặc biệt là kể từ khi Cục quản lý thuốc và thực phẩm Mỹ (US. FDA) phê duyệt thuốc Spritam- thuốc in $3 \mathrm{D}$ đầu tiên vào năm 2015. Công nghê in 3D cho phép chế tạo ra những vât thể thật theo đúng mô hình 3 chiều được thiết kế trên máy tính với độ chính xác cao. 
Trong các kỹ thuật in 3D, kỹ thuật tạo mô hình bằng bồi đắp-liên kết (fused deposition modeling - FDM) được đặc biệt quan tâm bởi khả năng ứng dụng rộng rãi và tiết kiệm hơn các kỹ thuật in 3D khác. Trong lĩnh vực khoa học dược, kỹ thuật này đã được ứng dụng để thiểt kế và bào chế nhiều dạng thuốc khác nhau, từ các thuốc giải phóng nhanh đến các thuốc giải phóng có kiểm soát. Đặc biệt, kỹ thuật này đã chứng tỏ được các ưu điểm vượt trội trong việc tạo ra các dạng bào chế phức tạp, gần như bất khả thi đối

\section{Nguyên tắc cấu tạo của máy in 3D FDM}

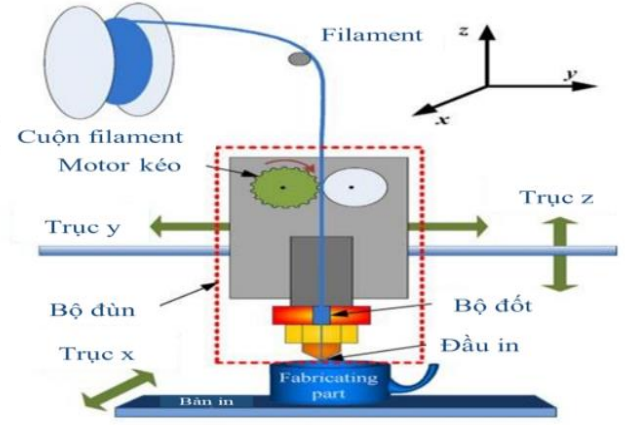

A với các kỹ thuật bào chế truyền thông. Thêm vào đó, kỹ thuật này đặc biệt phù hợp với mô hình chăm sóc y tế điện tử, khi mà thuốc được cá nhân hóa theo nhu câu điều trị của từng cá thể. Với mục tiêu tiếp cận và dần ứng dụng kỹ thuật in 3D trong lĩnh vực dược phẩm, bài viết này trình bày một cách có hệ thống nguyên lý cấu tạo, cơ chế hoạt động của các máy in 3D. Đồng thời, bài viết mô tả chi tiết từng bước quy trình tạo ra một vật thể bằng kỹ thuật in 3D dựa trên nguyên lý FDM.

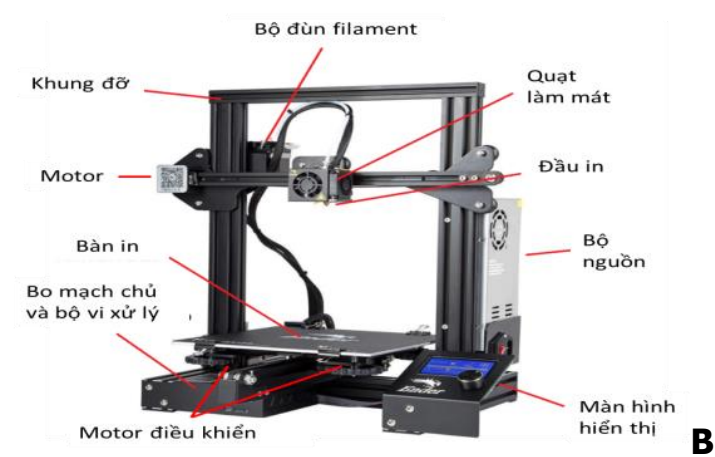

Hình 1: Sơ đồ nguyên tắc các bộ phận chính trong máy in 3D FDM (A) và cấu tạo của một máy in 3D FDM đơn giản (B)

Bàn in (đế in): là nơi lớp in đầu tiên dính kết, là giá đõ cho vật trong quá trình in.

Đầu in: Là nơi làm nóng chảy các sợi in và dẫn vật liệu in chảy lỏng bồi đắp lên lớp in trước với một tổc độ hẳng định.

Đùn filament: Là hệ thống gồm các bộ phận kéo, dẫn filament vào đâu in; bộ đốt nóng filament và đẩy mực in tạo các lớp trền bàn in.

Các Motors: Thường có 3 motor hoạt động độc lập phối hợp điều khiển chuyển động của đầu in và bàn in trên 3 trục $x, y, z$ theo hành trình đã được lập trình sẵn.

Bộ vi xử lý: Là trung tâm xử lý chuyển đổi các thông tin về cấu tạo của vật thể thành các lênh điều khiển bộ đùn filament, đâu in và bàn in. Bộ vi xử lý có thể trực tiếp điếu khiển máy in bằng cách lấy các dữ liệu về kết cấu từ file cắt lớp vật thể được lưu trong thẻ SD. Để có thể thực hiện các chức năng phức tạp hơn, bộ vi xử lý được kết nối và điều khiển bởi máy tính thông qua phần mềm đã được tích hợp sẵn. Việc điều khiển toàn diện bằng phần mềm trên máy tính cho phép thực hiện quá trình in linh động hơn, tạo ra các vật thể tinh xảo và có cấu trúc phức tạp hơn.

2. Hoạt động của máy in 3D dựa trên nguyên lý FDM.

FDM là quá trình làm nóng chảy vật liệu in, bồi đắp từng lớp để tạo thành cấu trúc chi tiết 3

chiều của vật thể. Quá trình này bao gồm các giai đoạn: 1) Tạo sợi in, "mực in 3D", từ các vât liệu thích hợp; 2) Thiết kế vật thể; 3) Chuyển định dạng tệp hình ảnh 3 chiều sang định dạng tệp chứa các thông tin về vị trí theo từng lớp; 4) Nạp nguyên liệu dưới dạng sợi in; 5) In vật thể theo mô hình; và 6) Hoàn thiện sản phẩm [2].

*Thiết kế mô hinh 3 chiều: Vật thể in được thiết kế hình dạng, kích thước, chi tiết cấu trúc bên trong trên các phần mềm thiết kế đồ họa 3 chiêu như AutoCAD, Sketchup hoặc máy quét 3 chiều (3D scanner).

*Chuyển định dạng file thiêt kế: Trước khi có thể in thành các vât thể, file đồ họ 3D phải được chuyển sang định dạng lớp bằng các phần mềm cắt lớp vật thể (slicing software). Các máy in 3D có thể thực hiện in trực tiếp từ file thiết kế theo lớp được nạp vào bộ xử lý của nó thông qua thẻ SD hoặc có thể được điều khiển bằng phần mềm in 3D được cài đặt trên máy tính. Sau khi tiếp nhận thông tin từ file thiết kế, bộ vi xử lý của máy in sẽ tính toán và thiết kế đường đi của đầu in cho mỗi lớp in.

*Tạo sợi in (filament): Trong bào chế thuốc bằng công nghệ in 3D FDM, tạo sợi in filament là bước đầu tiên và rất quan trọng. Nó quyết định chất lượng của sản phẩm và tính khả thi của quá trình in các dạng bào chế. Giai đoạn này cho 
phép phối hợp dược chất với các tá dược để tao ra "mực in" (hay filament). "Mực in" cho máy FDM là các sợi hình trụ, có đường kính đồng nhất, phù hợp với từng loại thiết bị in. Các máy in thượng mại thường được thiết kế để in các filament có đường kính $1,75 \mathrm{~mm}$ hoặc $2,85 \mathrm{~mm}$. Dược chất có thể được phối hợp vào các filaments bằng hai phương pháp đó là: thấm dược chất vào các filaments có sằn và đùn nóng chảy (hot melt extrusion).

- Phương pháp thứ nhất xuất phát từ các filaments đã được thương mại hóa. Đây là các sợi đùn được làm từ các nguyên liệu polymer (phổ biến là PBS, PLA, PVA...) và các chất phụ gia. Filaments thương mại được ngâm trong dung dịch dược chất được hòa tan trong dung môi thích hợp không có khả năng hòa tan các thành phần của filament thương mại [3]. Trong thời gian ngâm, dược chất sẽ khuếch tán vào trong filament. Sau khi đạt đến trạng thái cân bằng, filament được lấy ra và bay hơoi dung môi ở điều kiện thích hợp. Phương pháp này có ưu điểm là không cần trang bị một thiết bị chuyên dụng, đắt tiền để đùn sợi filament. Các filaments có độ đồng nhất cao, có kích thước và các tính chất cơ học thích hợp cho quá trình in. Tuy nhiên, phương pháp này có nhược điểm là chứa tỷ lệ dược chất rất thấp (thường nhỏ hơn $2 \%$ ) nên chỉ phù hợp cho các thuốc có liều dùng rất nhỏ [4]. Quá trình đưa thuốc vào sợi in tốn thời gian và có nguy cơ vượt giới hạn về tồn dư dung môi. Thêm vào đó, các nguyên liệu dùng để sản xuất sợi in có thể không được kiểm soát theo các quy định của sản xuất dược phẩm. Một trong những nhược điểm lớn của cách tiếp cận này là nhiệt độ in tương đối cao làm hạn chế khả năng áp dụng cho nhiều loại dược chất.

- Phương pháp thứ 2, xuất phát từ các nguyên liệu dược chất và các tá dược ở dạng bột, hat hoặc pellets. Hỗn hợp vật lý của công thức bào chế "mực in" được chuyển liên tục vào máy đùn nóng chảy để sản xuất các sợi in theo quy trình điển hình như mô tả dưới đây. Đùn nóng chảy là phương pháp được áp dụng phổ biến nhất trong các nghiên cứu ứng dụng kỹ thuật in 3D trong bào chế thuốc. Phương pháp này có ưu điểm là có thể thay đổi thành phần công thức bào chế linh động và dễ dàng, có thể áp dụng được với nhiều loại dược chất. Tỷ lệ dược chất trong filament cao, có thể lên đến $50 \%$ [5], mở ra khả năng áp dụng cho rất nhiều dược chất. Phương pháp đùn nóng chảy tiết kiệm thời gian, không sử dụng dung môi và dược chất ít tiếp xúc với môi trường, đồng thời có thể thực hiện tuân thủ các quy định GMP trong sản xuất dược phẩm. Việc xây dựng công thức bào chễ sợi in cần tính đến việc đảm bảo các tính chất cơ lý của filaments phù hợp với quá trình in 3D. Tính chất lưu biến của mực in nóng chảy cũng cần được tối ưu hóa để đảm bảo độ bền cơ học, tính chính xác với mô hình và độ phân giải phù hợp với các dạng bào chế. Quy trình sản xuất filament theo phương pháp đùn nóng chảy bao gồm các bước:

1) Tạo hỗn hợp vật lý của các nguyên liệu có trong cổng thức.

2) Cấp từ từ hỗn hợp vật lý vào máy đùn nóng chảy, tại đó nguyên liệu sẽ trở nên mềm hoặc nóng chảy dưới tác dụng của nhiệt tiếp tục được đồng nhất hóa và nén ép trong ông đùn (barrel) dưới tác động của trục vít.

3) Nguyên liệu nóng mềm (molten state) sẽ được trục vít đùn ép qua khuôn đặt ở cuối ống đùn có dạng một lỗ tròn với đường kính phù hợp để tạo ra các filament.

4) Các sợi đùn khi vừa ra khỏi khuôn còn ở có thể chất mềm, có thể kéo dãn, được chuyển liên tục trên một băng chuyền đồng bộ hóa với tốc độ đùn. Trong quá trình di chuyển trên băng chuyền, các sợi đùn được làm nguội dần, đông rắn tạo sợi in.

5) Các sơi đùn có thể được thu gom thành cuôn tròn bằng cách sử dụng thiết bi cuốn sơi. Sau đó, chúng được đóng gói và bảo quản cẩn thận trong đồ bao gói phù hợp.

* Các yêu cầu của filaments:

Các sợi in phải đồng nhất và có kích thước phù hợp với bộ phận dẩn - đẩy filament và đâu in của thiết bị. Đồng thời chúng cũng phải có bề mặt nhẵn, ít ma sát, độ cứng, khả năng đàn hội giúp filament đi vào đầu in dễ dàng, không bị biến dạng dưới lực đẩy của bộ đùn nhằm đảm bảo tính đồng nhất trong suốt quá trình in. Quan trọng hơn, mực in phải có nhiệt độ nóng chảy đồng rắn và tính chất lưu biến khi nóng chảy phù hợp nhằm giúp tạo liên kết tốt giữa các lớp in và đảm bảo độ chính xác về kết cấu, hình dạng sản phẩm [6]. Các tính chất và độ tương thích của filament với quá trình in $3 \mathrm{D}$ cần được đánh giá nhằm đảm bảo quá trình in được diển ra thông suốt [7].

*Chu trinh hoạt động của máy in 3D FDM

Để thực hiện quá trình in, trước tiên, bàn in phải được cân bằng và filament phải được nạp vào máy in thông qua một ống dẫn nhờ bộ phận đùn sợi được tạo bởi hai bánh răng quay ngược chiều nhau (Hình $1 \mathrm{~A}$ ). Khi hoàn tất việc cài đắt các thông số in như trình bày trong Bảng 1 , thiết 
bị in 3D sẽ khởi động và cần một khoảng thời gian để đạt cân bằng nhiệt độ của đầu in và bàn in. Sau thời gian ổn định, đầu in sẽ tự hạ thấp đến khi cách bàn in một khoảng đúng bằng chiều cao của mỗi lớp in, sẵn sàng cho việc in lớp đầu tiên.

\begin{tabular}{|c|c|c|}
\hline $\mathbf{T}$ & Thông số & Ý nghĩa của thồng số \\
\hline 1 & $\begin{array}{c}\text { Tốc độ cấp sợi in (Feeding } \\
\text { speed) }\end{array}$ & $\begin{array}{c}\text { Cài đặt tốc độ đùn sợi in, được đồng bộ hóa với tốc độ di } \\
\text { chuyển của đâu in. }\end{array}$ \\
\hline 2 & $\begin{array}{l}\text { Tốc độ rút filament (Filament } \\
\text { retraction speed) }\end{array}$ & $\begin{array}{c}\text { Cài đặt tốc độ rút filament khi đầu in gặp khoảng trống } \\
\text { trong mô hình vật in. }\end{array}$ \\
\hline 3 & $\begin{array}{l}\text { Mức độ rút filament (Filament } \\
\text { retraction) }\end{array}$ & $\begin{array}{l}\text { Cài đặt mức độ rút filament khi đầu in đến khoảng trống } \\
\text { trong mô hình hoặc thay đổi mực in. }\end{array}$ \\
\hline 4 & $\begin{array}{l}\text { Kích thước filament (Filament } \\
\text { diameter) }\end{array}$ & $\begin{array}{c}\text { Cho phép tùy chỉnh theo đường kính thực tế của filament đế } \\
\text { đồng bộ hóa với tốc độ in }\end{array}$ \\
\hline 5 & $\begin{array}{l}\text { Nhiệt độ in (Print head } \\
\text { temperature) }\end{array}$ & $\begin{array}{l}\text { Cài đă̆t nhiệt độ của đầu kim loại nơi làm nóng và chuyển } \\
\text { mực in từ trạng thái rắn sang trạng thái chảy lỏng. }\end{array}$ \\
\hline 6 & $\begin{array}{l}\text { Nhiệt độ bàn in (Platform } \\
\text { temperature) }\end{array}$ & $\begin{array}{c}\text { Cài đặt nhiệt độ của bề mặt bàn in, nơi lớp in đầu tiên tiếp } \\
\text { xúc. }\end{array}$ \\
\hline 7 & $\begin{array}{l}\text { Tốc độ di chuyển đầu in } \\
\text { (Print head velocity) }\end{array}$ & theo chiều dài hành trình của đâu in \\
\hline 8 & Tốc độ quạt (Fan speed) & Cài đặt tốc độ quạt làm mát bề mặt vừa in. \\
\hline 9 & $\begin{array}{c}\text { Mấu hình mồi lớp in (Print } \\
\text { pattern) }\end{array}$ & $\begin{array}{l}\text { Cài đặt dạng hành trình của đâu in trên mối lớp in, có thể } \\
\text { vòng hướng tâm, đường zic zac, hình caro ... }\end{array}$ \\
\hline 10 & Độ lấp đầy sản phẩm (Infill) & $\begin{array}{l}\text { Quy định \% không gian bị chiếm chố bởi nguyên liệu in ở } \\
\text { bên trong vật thể. }\end{array}$ \\
\hline 11 & $\begin{array}{l}\text { Độ dày lớp bao ngoài vật in } \\
\text { (Shell thickness) }\end{array}$ & Bề dày lớp bao đặc khít ngoài cùng của vật thể. \\
\hline 12 & $\begin{array}{l}\text { Độ dày lớp in (Layer } \\
\text { thickness) }\end{array}$ & Độ dày, của mỗi lớp in, quy định độ phân giải. \\
\hline 13 & $\begin{array}{c}\text { Góc nghiêng đường in (Raster } \\
\text { orientation) }\end{array}$ & Góc tạo bởi dường in thẳng và truc x của hệ tọa độ in. \\
\hline 14 & $\begin{array}{l}\text { Góc nghiêng vật thế khi in } \\
\text { (Build orientation) }\end{array}$ & $\begin{array}{c}\text { Cho phép điều chỉnh độ nghiêng của vật so với mặt phằng } \\
\text { của bàn in }\end{array}$ \\
\hline
\end{tabular}

Khi nhiệt độ đầu in đạt giá trị cài đặt, quá trình in bắt đâu với sự chuyển động ngược chiều nhau của 2 bánh răng trong bộ phận đùn giúp kéo filament vào máy in. Tại phần kim loại của đầu in, filament được làm nóng chảy. Dung dịch nóng chảy được đẩy qua đầu in kim loại tạo các lớp trên khay in với độ dày lớp in có thể cài đặt

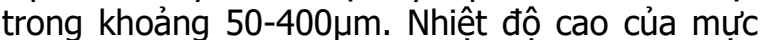
in làm bề mặt lớp in cũ chảy lỏng và giúp kết dính với lớp in mới. Chúng sẽ nhanh chóng đông rắn lại ở nhiệt độ thường hoặc có thêm sự trợ giứp của quạt làm mát (Hình $2 A$ ). Khi kết thúc mỗi lớp in, đầu in sẽ được nâng lên hoặc bàn in sẽ được hạ xuống một khoảng bằng chiều dày lớp in đã được cài đặt, sẵn sàng cho việc bồi đắp lớp in mới trền phần đã được in. Quá trình cứ thế lặp lại cho đến khi tạo thành vật thể hoàn chỉnh.

Trong phần lớn các máy in 3D thương mại, đầu in được có thể di chuyển trên mặt phẳng song song với bàn in theo 2 trục y và $z$ trong khi bàn in có thể di chuyển theo trục $x$ thông qua sự truyền động bằng dây đai từ các động cơ. Tuy nhiên, cũng có những loại máy cố định đầu in có thể di chuyển trên mặt phẳng song song với bàn in theo hai trục $x, y$ trong khi khay in có thể di chuyển độc lập theo phương thẳng đứng vuông góc với bàn in (trục $\mathrm{z}$ ). Các thiết bị in $3 \mathrm{D}$ truyền thống chỉ có một đầu in, trong khi các máy in 3D hiên đai có thể có 2 đâu in hoăcc hơn. Đầu in phổ biến là đầu in đơn chỉ có thể đùn một loại filament trong quá trình in (hình $2 A$ ).
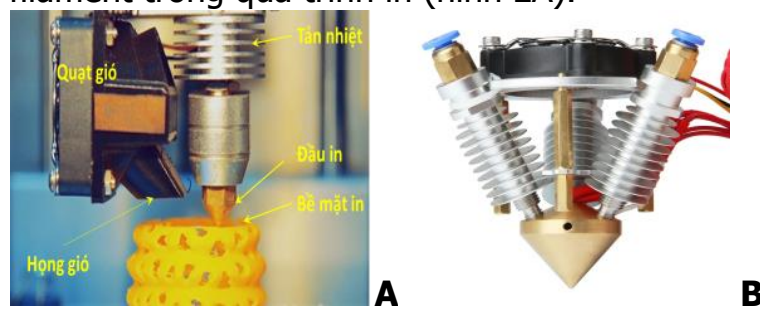

Hình 2: Cấu tạo của đầu in và quạt làm mát $A$ ) Đầu in đơn và quạt làm mát lớp in; B) Đầu in kép có thể in đờng thời nhiều filaments 
Trong khi đó, ít phổ biến hơn, đầu in kép có thể đùn hai hay nhiêu loại filaments riêng biệt, luân phiên hoặc đồng thời trong quá trình in (hình 2B). Nhờ vậy, có thể tách biệt mực in có chứa các nguyên liệu tương ky hoặc cho phép in vật thể có nhiều phần mà mỗi phần được tạo thành từ các nguyên liệu khác nhau.

*Hoàn thiên sản phẩm: Trong một số trường hợp, vật thể in có hình dạng phức tạp hoăcc diên tích bề mặt in nhỏ khiến cho in lớp đâu tiên khó khăn hoặc vật thể in không định hình vững chắc trên bàn in. Trong trường hợp này, có thể tạo các khung đõ định hình cấu trúc của vật hoặc hỗ trợ các lớp đầu liên kết với bề mặt bàn in. Những vật liệu định hình này sẽ được loại bỏ sau khi quá trình in kết thúc. Tùy thuộc vào nguyên liệu cấu thành, vật liệu trung gian có thể bị loại bỏ bằng cách bẻ gãy hoặc hòa tan trong nước ấm [8]. Bên cạnh vệ sinh loại bỏ các vật liệu trung gian, giai đoạn hoàn thiện có thể gồm công đoạn thêm những đặc tính mới cho sản phẩm, như đánh bóng, bao film, bao màu...

\section{KẾT LUÂ̂N}

Cơ chế hoạt động của kỹ thuật in 3D FDM tương đối đơn giản, dễ điều khiển và có thể kiểm soát hoàn toàn bằng phần mềm. Kỹ thuật này cho phép tạo ra các vật thể có cấu trúc phức tạp với độ chính xác cao từ các mô hình 3 chiều. Đặc tính của vật in có thể được điều chỉnh linh hoạt thông qua điều chỉnh thiết kế của vật in cũng như thay đổi các thông số điều khiển quá trình in. Việc hiểu rõ nguyên lý cấu tao, quy trình hoạt động là rất cần thiết trong việc làm chủ công nghệ và kiểm soát quá trình hoạt động nhằm đảm bảo chất lượng sản phẩm tốt và độ lặp lại cao. Công nghệ này mở ra hướng hiện đại hóa ngành công nghiệp sản xuất thuốc theo hướng tạo ra các dạng thuốc có cấu trúc phức tạp và cá nhân hóa điều trị trong chăm sóc y tế.

TÀI LIỆU THAM KHẢO

1. Katstra, W., Palazzolo, R., Rowe, $C_{\text {., }}$ Giritlioglu, B., Teung, P., and Cima, M. (2000). Oral dosage forms fabricated by Three Dimensional Printing ${ }^{\mathrm{TM}}$. Journal of controlled release $66,1-9$.

2. Gaisford, A.W.B.a.S. (2018). 3D printing of pharmaceuticals.

3. Goyanes, A., Buanz, A.B., Hatton, G.B., Gaisford, S., and Basit, A.W. (2015). 3D printing of modified-release aminosalicylate (4-ASA and 5-ASA) tablets. European Journal of Pharmaceutics and Biopharmaceutics 89, 157-162.

4. Goyanes, A., Buanz, A.B., Basit, A.W., and Gaisford, S. (2014). Fused-filament 3D printing (3DP) for fabrication of tablets. International journal of pharmaceutics 476, 88-92.

5. Gioumouxouzis, C.I., et al. (2018). "A 3D printed bilayer oral solid dosage form combining metformin for prolonged and glimepiride for immediate drug delivery.". European Journal of Pharmaceutical Sciences, 120, 40-52.

6. Aho, J., et al. (2019). "Roadmap to 3D-printed oral pharmaceutical dosage forms: feedstock filament properties and characterization for fused deposition modeling." Journal of pharmaceutical sciences, 108, 26-35.

7. Xu, P., et al. (2020). "Development of a quantitative method to evaluate the printability of filaments for fused deposition modeling 3D printing.". International journal of pharmaceutics, $588,119760$.

8. Gebhardt, A., and Jan-Steffen Hötter. (2016). Characteristics of the Additive Manufacturing Process, Additive Manufacturing D, 3, 21-91.

\section{HIỆU QUẢ CHE TUỶ TRỰC TIẾP BẰNG VÂTT LIỆU CALCIUM SILICATE (BIODENTINETM) TRÊN RẲNG VĨNH VIỄN CÓ VIÊM TUỶ KHÔNG HỒI PHỤC}

\section{TÓM TĂT}

Mục tiêu: Đánh giá hiệu quả che tuỷ trực tiếp bằng vật liệu calcium silicate (Biodentine ${ }^{T M}$ ) trển răng người trưởng thành có viêm tuỷ không hôi phục. Đối tượng và phương pháp nghiên cứu: Bênh nhân nữ 24 tuổi có cơn đau tự phát, kéo dài, xuất hiện

*Đai Hoc Y Dước Tp.Hồ Chí Minh

Chịu trách nhiệm chính: Trần Xuân Vĩnh

Email: vinhdentist@yahoo.com

Ngày nhận bài: 13.4.2021

Ngày phản biên khoa hoc: 27.5 .2021

Ngày duyệt bài: 14.6.2021

\section{Trần Xuân Vĩnh*}

nhiều lần cách đây 2 tuần. Dựa trên khám lâm sàng và hình ảnh $X$ quang, răng được chẩn đoán viêm tuỷ không hồi phục. Sau khi gây tê và đặt đê cao su, phân ngà sâu và mô tuỷ viêm được loại bỏ bằng mũi khoan vố trùng và tay khoan cao tốc dưới nguồn nước. Cầm máu trong vòng 2 phút bằng gòn thấm $\mathrm{NaOCl} 2,5 \%$. Tuỷ lộ được che bằng vật liệu Biodentine ${ }^{T M}$. Sau 1 tháng, răng được trám kểt thúc với resin composite. Kết quả: Ngày đâuu tiên sau can thiêp, bênh nhân chỉ đau nhẹ. Theo dõi sau 1 tháng, 6 tháng và 24 tháng, răng không có triệu chứng bất thường, đáp ứng với thử nhiệt hoặc điện. Hình ảnh trên phim $X$ quang vùng quanh chóp bình thường. Kêt luận: Che tuỷ trực tiếp với vật liệu Biodentine ${ }^{T M}$ trên răng trưởng 